\title{
ESPECIALIZACIÓN Y DIVISIÓN DEL TRABAJO EN LA PROFESIONALIZACIÓN DEL SECTOR SIN FINES DE LUCRO
}

\author{
ESPECIALIZAÇÃO E DIVISÃO DO TRABALHO NA \\ PROFISSIONALIZAÇÃO DO SETOR SEM FINS-LUCRATIVOS
}

\section{SPECIALIZATION AND DIVISION OF LABOR IN THE NON-PROFIT SECTOR PROFESSIONALIZATION}

\author{
Marina Melo ${ }^{1}$ \\ https://orcid.org/0000-0003-4402-4680 \\ Lorena Madruga Monteiro ${ }^{2}$ \\ https://orcid.org/0000-0002-3720-7684
}

Submissão: 16/06/2021 / Aceito: 29/07/2021 / Publicado: 04/02/2022.

\begin{abstract}
Resumen
El presente estudio investiga cuáles son las consecuencias del actual flujo de profesionalización institucional de las ONG en Brasil. La profesionalización se trata de estrategias de planificación y especialización. En cumplimiento de nuestra pregunta inicial, analizamos aspectos como: división del trabajo; existencia de diferentes tipos de ONG; visión de los agentes que trabajan en las instituciones ante el actual flujo de profesionalización; misión de las organizaciones ante los requerimientos de profesionalización por parte de las agencias financiadoras; relación entre la misión y la sostenibilidad financiera de las entidades; así como la relación que las ONG tienen con el Estado, con el Mercado y con el propio tercer sector, considerando la extensión interviniente que atañe al tema de la autonomía institucional. La investigación se basó en la Sociología de las Organizaciones, además de adoptar métodos y técnicas de trabajo en una perspectiva cualitativa, a través de estudios de caso en dos organizaciones, que utilizaron el análisis de contenido en el tratamiento de la información. El trabajo revela cómo y por qué las ONG que ni siquiera se adaptan mínimamente a los requisitos de profesionalización se desvanecen ante las fuentes de financiación. La búsqueda de la sostenibilidad y, en consecuencia, de la complejidad organizativa dentro de algunos de los perfiles discutidos de las ONG en el trabajo genera un ciclo de problemas que solo se pueden resolver con más profesionalismo. Vinculado a este escenario, la agencia del tercer sector es relativamente frágil al amplio proceso de profesionalización, habitando una "nube" de inseguridad sobre la misión institucional a la que se dedica.
\end{abstract}

Palabras clave: ONGs; Tercer sector; profesionalismo; financiamiento, Especialización.

\footnotetext{
${ }^{1}$ Doutora em Sociologia pela UFPE. Professora Adjunta Permanente do Instituto de Ciências Sociais da Universidade Federal de Alagoas (UFAL).E-mail. melomarina@msn.com

${ }^{2}$ Doutora em Ciência Política pela UFRGS. Professora do Programa de Pós-Graduação em Sociedade, Tecnologias e Políticas Públicas (SOTEPP) do Centro Universitário Tiradentes (UNIT/AL). E-mail. lorena.madruga@gmail.com
} 


\title{
Resumo
}

O presente estudo investiga quais as consequências do atual fluxo de profissionalização institucional das ONGs no Brasil. Profissionalização diz respeito a estratégias de planejamento e especialização. Em cumprimento a nossa questão de partida, analisamos aspectos como: divisão de trabalho; existência de diferentes tipos de ONGs; visão dos agentes que nas instituições trabalham diante do atual fluxo de profissionalização; missão das organizações face exigências de profissionalização por parte dos órgãos financiadores; relação entre a missão e a sustentabilidade financeira das entidades; bem como a relação que as ONGs têm com o Estado, com o Mercado e com o próprio terceiro setor, a considerar a extensão interveniente que diz respeito à questão da autonomia das instituições. A pesquisa apoiou-se na Sociologia das Organizações, bem como adotamos métodos e técnicas de trabalho numa perspectiva qualitativa, por estudos de caso em duas organizações, que utilizou a análise de conteúdo no tratamento das informações. O trabalho revela como e porque ONGs que não se adequam minimamente às exigências de profissionalização esmaecem diante das fontes de financiamento. A busca por sustentabilidade e, consequentemente, por complexidade organizacional dentro de alguns perfis de ONGs no trabalho discutidos, gera um ciclo de problemas que só podem ser solucionados com mais profissionalização. Concatenada a este cenário, a agência do terceiro setor apresenta-se relativamente frágil ao processo amplo de profissionalização, habitando uma "nuvem" de insegurança sobre a missão institucional para a qual se dedica.

Palavras-Chave: ONGs; Terceiro Setor; Profissionalização; Financiamento, Especialização.

\begin{abstract}
The present study investigates what are the consequences of the current flow of institutional professionalization of NGOs in Brazil. Professionalization means planning and specialization strategies. In fulfillment of our initial question, we analyzed aspects such as: division of labor; existence of different types of NGOs; view of agents who work in institutions in the face of the current flow of professionalization; mission of organizations in the face of professionalization requirements on the part of funding agencies; relationship between the mission and the financial sustainability of the entities; as well as the relationship that NGOs have with the State, with the Market and with the third sector itself, considering the intervening extension that concerns the issue of institutional autonomy. The research was based on the Sociology of Organizations, as well as adopting work methods and techniques in a qualitative perspective, through case studies in two organizations, which used content analysis in the treatment of information. The work reveals how and why NGOs that are not even minimally suited to professionalization requirements fade in the face of funding sources. The search for sustainability and, consequently, for organizational complexity within some of the discussed profiles of NGOs at work generates a cycle of problems that can only be solved with more professionalism. Linked to this scenario, the third sector agency is relatively fragile to the broad process of professionalization, inhabiting a "cloud" of insecurity about the institutional mission to which it is dedicated.
\end{abstract}

Keywords: NGOs; Third Sector; Professionalization; funding organizations, Specialization. 


\section{APRESENTACIÓN}

Las siguientes páginas tienen como objetivo central analizar empíricamente las consecuencias del actual flujo de profesionalización institucional de las ONG brasileñas a partir de las dimensiones de la especialización y de la división del trabajo. Se observa que la profesionalización institucional ha sido un elemento clave en las actuales configuraciones del tercer sector, siendo un fenómeno amplio y que debe ser investigado en sus ejes analíticos centrales cuando deseamos entender específicamente la lógica de funcionamiento del sector sin fines de lucro. Por una etnografía, buscamos contribuir a un debate más general sobre profesionalización de entidades del tercer sector a ver como ha ocurrido el proceso de profesionalización en las ONGs. Aunque muchos trabajos han mencionado la profesionalización en el tercer sector como un fenómeno contemporáneo en atención (LANDIM, 1993; CARVALHO, 1999; HADDAD, 2002; LIMA, 2004; COSTA, 2004, LIMA, 2020; MELO, 2020; PAES, 2019; TENÓRIO, 2015; VIEIRA, 2020), observamos que su aspecto en esas instituciones, cuando se considera junto a la cultura organizacional de las entidades y cómo éstas tratan con las transformaciones, no sólo en el universo del tercer sector, sino también del mundo del trabajo visto desde una óptica panorámica, aún no ha sido explorado de modo más específico por la literatura sobre ONGs, aunque algunos autores apuntan a la importancia de la temática y proveen subsidios para ese tipo de estudio. En este sentido, nuestra propuesta es la de fomentar tal dimensión, estableciendo nuevas relaciones que puedan enriquecer, problematizar, relativizar o confirmar trabajos anteriores.

Las principales categorías consideradas en este trabajo para caracterizar la profesionalización institucional de las ONGs son: recorrido escolar y grado de escolaridad de los agentes que trabajan en la organización; nivel de especialización en las actividades desarrolladas; división de tareas administrativas; interacción entre las relaciones primarias y secundarias de sociabilidad en el trabajo; capacidad de articulación de las entidades en redes de cooperación; estrategias y planificación institucional y dinámica de evaluación del trabajo interno. Estas dimensiones no son excluyentes, ni tampoco exhaustivas.

Optamos por utilizar como base teórica principal para la problematización expuesta la sociología de las organizaciones y sus contribuciones en un enfoque centrado en el fenómeno organizacional y en la división del trabajo en las ONGs. Tal perspectiva analiza cómo las instituciones se disponen ante un contexto globalizado que tiene sistemas de 
gerencia contrastantes y que acarrean en diferentes formas de planificar y realizar tareas. Las diferentes culturas organizacionales - entendidas como la manera en que las ONGs estructuran sus formas de actuación, de organización y cómo esbozan los medios de ejecutar los trabajos - pasan por deficiencias en los planes administrativos, principalmente cuando no están insertas en una lógica de profesionalización que viabilice ejecución de los proyectos de la institución. Estos principios, como escriben Hill y Egan (1967), dependen de dimensiones analíticas que nos ayudan a percibir la profesionalización de una entidad. Mientras tanto, estas dimensiones se reelaboran en diferentes sistemas de gestión y contextos. "Estas decisiones dependen en gran medida de la amplia diversidad de las variables de trabajo, así como de los objetivos que estructuran la organización, por lo que las proposiciones administrativas sólo pueden expresarse en términos de una situación particular". (HILL, EGAN, 1967, p. 332).

Las motivaciones individuales pueden incluso interferir en el funcionamiento y la gestión de una entidad, sin embargo, la rigidez del modelo organizacional propuesto por los agentes involucrados en las $\mathrm{ONG}$ es un factor determinante para la conducción de esas influencias. Lakatos (1987) no explica que una organización, también, se refiere a conductas humanas que se relacionan socialmente y se basa en las regularidades (LAKATOS, 1987, p. 228). Selznick (1949) refuerza que considerar la simple creación de una estructura formal como una organización no sostiene a esta organización como tal, pues, es necesario que sea creada a partir de necesidades colectivas, ya que es más que una agrupación de personas (LAKATOS, 1987, p. 228). Una organización tiene identidad propia y puede ser considerada como una unidad social intencionalmente elaborada. Por lo tanto, no podríamos decir que una organización es simplemente el edificio donde está localizada, incluso porque muchas no poseen tal formato físico/estructural. Igualmente, no podríamos también decir que lo que define si una ONG es más o menos profesionalizada es el tamaño de sus instalaciones, pues algunas ONGs pueden ser virtuales, lo que genera, a su vez, un debate paralelo y relevante sobre las redes sociales en tercer sector. Por lo que decimos, utilizamos la sociología de las organizaciones a partir del contexto más específico de las organizaciones de tercer sector actualmente. Llegamos a la necesidad de cuestionar la agencia ante un estudio más concentrado en la estructura. Explicamos: si la sociología de las organizaciones funcionó como guía principal en la conducción de la investigación, por otro lado, no pudimos desconsiderar que aunque el foco de la propuesta de investigación 
fue el estudio de la profesionalización de las ONGs, estas últimas son formadas por agentes que las hacen funcionar y estos agentes (la forma en que se ocupan de las organizaciones y de sus trabajos) pasaron a ser una dimensión analítica de investigación. En otras palabras, sería inviable intentar entender cómo funcionan las organizaciones sin considerar a sus agentes.

Utilizamos también como auxiliar teórico la sociología de las profesiones. A pesar de que la sociología de las profesiones ser una guía para nuestro análisis, no la utilizamos como una teoría cerrada en sí capaz de contestar la realidad de nuestro objeto de estudio. Nuestro esfuerzo se ha emprendido en la percepción de esa construcción elaborada junto al estudio de cómo se dan los diseños organizacionales en las instituciones, es decir, cómo se formatean las profesiones y sus funciones dentro de una lógica más grande de gestión de las entidades. Asimismo, tratamos del voluntariado como clave-analítica en el entendimiento de las condiciones en que se establecen las cuestiones tratadas de la estructura de las organizaciones frente a las interacciones de una agencia representativa de diversos papeles sociales de trabajo.

Finalmente, explicamos que, luego de esta breve presentación, este artículo continuará por la siguiente organización: 2. Sobre la metodología utilizada; 3. Retrato de las fundaciones y asociaciones sin fines de lucro en Brasil; 4. Presentación de las Organizaciones No Gubernamentales; 5. Divisiones de Trabajo, Especialización y Búsqueda por Profesionalización; 6. Aspectos que caracterizan un escenario en movimiento y, 7. consideraciones finales.

\section{SOBRE LA METODOLOGÍA UTILIZADA}

$\mathrm{Si}$, desde una perspectiva amplia, optamos por realizar una investigación cualitativa, en una dimensión más restringida, adoptamos el análisis de contenido como técnica de procesamiento de información, sustentada esencialmente por Bardin (1977) a partir de su caracterización más general y sistemática, que puede trabajar con una cantidad variada de materiales y que se centra en el objeto que es el contenido a analizar (manifiesto o latente). Sus principales procedimientos son la codificación, categorización e inferencias.

La primera fase se refiere a la operacionalización del material a estudiar y la sistematización de ideas. Sus principales pasos son: lectura exploratoria; la elección de documentos; la preparación del material; la referenciación de índices y la elaboración de 
indicadores. En nuestra investigación, esta primera fase se desarrolla en dos instancias, una, para ser presentada al lector a lo largo del enfoque de las ONG con las que trabajamos en campo, y otra, más "backstage", que es la fase inicial de la investigación. incluso antes de la organización del material a tratar, es decir, antes del propio análisis de contenido. Por ello, en un primer momento realizamos una revisión bibliográfica de estudios sobre el tercer sector, en particular, de títulos referentes al fundamento teórico de esta investigación. Con base en un criterio de división temática, los textos leídos y sus respectivas problematizaciones fueron anotados y agrupados según temas relevantes a las ideas presentadas a lo largo de esta investigación. Así, sistematizamos las lecturas en formas especiales conteniendo títulos como: sociología de las organizaciones, sociología de las profesiones, autonomía, profesionalización y especialización en estas organizaciones y otras entradas relacionadas con el objeto de estudio. Contamos con apoyo teórico y metodológico, por ejemplo, para avanzar en la selección de documentos que fueron analizados en las instituciones y para realizar métodos y técnicas de investigación desde un análisis temático dentro del análisis de contenido más general.

La segunda fase propuesta por Bardin, exploración del material, involucró el análisis de unidades de registro y unidades de contexto. En cuanto a los instrumentos de recolección y procesamiento de información, el presente trabajo se realizó, fundamentalmente, con los recursos de observación participante y directa; entrevistas semiestructuradas y análisis de documentos.

Con este inicio del trabajo de campo, a través de observaciones directas y participativas, pudimos elaborar preguntas más relacionadas con las realidades de las ONG, así como tener un mejor acceso a los agentes para pasar a la etapa de recolección de información, a través de la cual realizamos 37 entrevistas semi-estructuradas.

También como herramienta de recopilación de información, desarrollamos un análisis de documentos, que es "una operación o conjunto de operaciones destinadas a representar el contenido de un documento en una forma diferente a la original, con el fin de facilitar la consulta y referenciación en un estado posterior" (BARDIN, 1977). El análisis documental está contenido en el análisis de contenido del que hablaremos a continuación, es decir, en el proceso de codificación de las fuentes de financiamiento de las ONG. Por lo tanto, la investigación se basa en el examen de documentos de las instituciones, como informes de actividades, reuniones agendas, objetivos perseguidos y/o logrados por las 
organizaciones. El análisis documental también estuvo guiado por artículos de periódicos, revistas u otras fuentes publicadas sobre las instituciones. A la mayor brevedad, los textos producidos por los agentes fueron utilizados y utilizados por estos con fines didácticos, entre otros fines. Este tipo de material se puede encontrar en ONG y, a menudo, en sitios web, libros y folletos informativos.

\section{RETRATO DE LAS FUNDACIONES PRIVADAS Y ASOCIACIONES SIN FINES DE LUCRO EN BRASIL}

Por la preocupación con las dimensiones que toman nuestro objeto de estudio, utilizamos datos secundarios para mapear a las ONGs en Brasil en algunos aspectos que, aunque no sean foco exclusivo al objetivo del presente trabajo, se hacen necesarios y nos auxilian a contemplar el tercer sector en una lupa macro, nacional brasileña y cuantitativa, antes de considerar sus especificidades en un plano más cualitativo y estrecho. Recurrimos a la investigación "As fundações privadas e as associações sem fins lucrativos no Brasil", (2016) que registró cerca de 602 mil entidades privadas sin fines de lucro en Brasil, teniendo en cuenta entidades diversas, como las que no encajan en el perfil de ONGs, de acuerdo con nuestro trabajo. Al analizar sólo las que considera FASFIL (fundaciones y asociaciones sin fines de lucro), llega a un número de 338,2 mil fundaciones y asociaciones, que representaban cerca del 5,6\% del total de instituciones privadas lucrativas, privadas no lucrativas y públicas de Brasil. De acuerdo con la encuesta, el 42,4\% de esas asociaciones y fundaciones están ubicadas en el Sudeste de Brasil, donde hay un equilibrio entre la cantidad de entidades y el tamaño poblacional. La región Nordeste, que posee cerca del $27 \%$ de la población brasileña, posee el 23,7\% de las instituciones. Es en la región sur del País donde hay la mayor concentración de organizaciones teniendo en cuenta el tamaño de la población sureña, que es del 14,6\% y posee el $22,7 \%$ de las entidades. Por otro lado, la región norte es donde se verifica mayor desproporción de esa lógica de equilibrio poblacional versus cantidad de organizaciones, ya que cuenta con cerca del $8 \%$ de la población nacional y con apenas el 4,8\% de entidades. La investigación ratifica además que estas organizaciones son relativamente nuevas: mayoría fundada en la década de 1990, siendo el 41,5\% de esas, recientes, ONGs orientadas a la promoción del desarrollo y defensa de derechos e intereses de los ciudadanos, a incluirse allí, igualmente, las 
asociaciones empresariales y profesionales. (IBGE ${ }^{3}, 2016$, p. 24). Las nacidas antes de 1980 corresponden a sólo el 13,1\% de las encuestadas, lo que indica, de acuerdo con el informe, un ritmo de disminución del nacimiento de entidades de la época, teniendo en cuenta los problemas que éstos tienen de sostenibilidad.

En las áreas específicas de actuación de las FASFIL, o sea, de las ONGs, se nota que la presencia religiosa en la formación de las entidades es relevante, considerando que un cuarto de las entidades presenta conexión al tema. Sin embargo, se considera el problema de muchas instituciones no ser a la religión relacionadas porque, aunque amparadas por ideologías católicas, protestantes o afro-brasileñas, por ejemplo, no son así denominadas en sus misiones, lo que generó un problema de catalogación a los investigadores del IBGE (¿como categorizar pesos religiosos en el propuesto modelo de investigación?). Otro punto es que, aún las ONGs detectadas por el IBGE como notadamente relacionadas a la religión, no se expresa en la investigación cuáles las religiones, lo que no podríamos, habiendo visto los diferentes formatos de sincretismo brasileño, saber si hay una proporción correlata entre las representaciones religiosas en el País y esas manifestaciones en el ambiente del Tercer Sector.

En las ONGs brasileñas, se observan que los cambios circundantes al desarrollo de las entidades están vinculados a la fecha de fundación de las organizaciones (IBGE, 2016, p. 29), sobre todo porque nacidas en contextos de oportunidades, visiones y modelos de gerencias varios. En las antiguas, es decir, creadas antes de 1980, predominan instituciones con vertientes religiosas, del área de cultura y recreación y de asistencia social. En 2005, sin embargo, tal representación cambia, decayendo el número de instituciones nacidas de vertientes religiosas, a crecer el número de ONGs de defensa de derechos de los ciudadanos que a la altura ya representaba más del $40 \%$ del total de instituciones brasileñas (IBGE, 2016).

Sobre la empleabilidad en el Sector, las FASFIL investigadas dirigen, formalmente por las leyes de trabajo, 1.7 millones de personas en Brasil. Por comparación, esto significaría alrededor del $22 \%$ del total de empleos públicos del País a considerar que los empleos públicos son formalizados, registrados como tales, y el 22\% del que hablamos son sólo parte del universo del Tercer Sector, puesto que todo el voluntariado no se incluye en esa categoría de trabajadores asalariados. $57.1 \%$ de las ONGs que tienen algún empleo

\footnotetext{
${ }^{3}$ Instituto Brasileiro de Geografia e Estatística. 
formal se localizan en el Sudeste de Brasil. Sólo la provincia de São Paulo concentra 553,7 mil trabajadores asalariados en ONG (es decir, el 34.4\% de los asalariados están ahí). Estas cifras no acompañan el desarrollo nacional de empleos, que concentra alrededor del $22 \%$ de los empleos en São Paulo, y no un número superior a 34, como se ve en el universo del trabajo formal en ONGs. Si, por un lado, consideramos la concentración de esos empleos por región, por otro, percibimos que ella también ocurre en función de las áreas de actuación de las entidades. Un ejemplo a corroborar con esa información es que el sector de educación e investigación de las ONGs, que representa sólo el 5,9\% de las entidades brasileñas analizadas, emplea el $29,8 \%$ de los trabajadores formales del tercer sector en el País (IBGE, 2016).

Uno de los supuestos que sostiene la imagen de que Brasil está compuesto, en su mayoría, de ONGs de pequeño porte es que el 79,5\% de las entidades del País no poseen ningún empleado formalizado. Este elevado porcentaje se acentúa cuando miramos a las Regiones Nordeste y Norte exclusivamente, llegando al $87,9 \%$ y el $84,4 \%$, respectivamente. Se supone, consecuentemente, que son el voluntariado y el trabajo informal que apoyan la mayoría de las entidades brasileñas, especialmente, de las dos regiones citadas.

La investigación explica además que no sólo la región se refiere al desequilibrio de entidades sobre el número de asalariados que poseen, pero también que el tiempo de surgimiento de esas instituciones suena como una variable imprescindible en la descripción de tal escenario, a tener en cuenta que si aislamos el pequeño grupo de 2.932 instituciones, que constan con más de 100 asalariados cada una, tenemos que el 58,6\% de ellas surgieron antes de 1980 (IBGE, 2016, p. 39), o sea, son las ONGs antiguas que cargan las estructuras de mayor porte, de acuerdo con las consideraciones del IBGE sobre el porte de las instituciones.

Los salarios provenientes del tercer sector brasileño mueven cerca de 24 mil millones de reales brasileños al año, aproximadamente 7,3 mil millones de dólares (2018). Sin embargo, una vez más consideramos las diferencias entre las regiones analizadas y vemos que la media de salarios de la región Nordeste, 2,7 salarios mínimos locales, es inferior a la media nacional, de 3,8 salarios mínimos. Se observa que esta diferencia de promedio salarial no sólo se da en el tercer sector, sino también en el sector público, que tiene un promedio salarial en el país de 3,7 salarios mínimos y, en el Nordeste, de apenas 
2,7 salarios (IBGE, 2016, p. 43). Consideremos también que estas diferencias estructurales forman parte de un modelo económico/financiero más amplio porque la baja de salarios estimula un costo de vida menos pesado que, aunque no justifique las disparidades numéricas encontradas, nos hace tener en cuenta que no podemos comparar, por ejemplo, el costo de vida de una ciudad como São Paulo-SP, sudeste, al costo de la vida de una ciudad como João Pessoa-PB, en el nordeste del País.

Sobre el porte de las instituciones para el IBGE, en algunos sectores, en particular los de 'asistencia social' y 'cultura y recreación', sus portes vienen decreciendo desde 1996. A ello, los datos no responden, por sí, cuáles los impactos que esa caída numérica (de funcionarios por organización) causa en el tercer sector, tampoco sobre su implicación en la calidad de los servicios prestados y en la cobertura de dichos servicios. Igualmente, esos datos no responden sobre cómo ha sido (o no) la sustitución del cuadro operativo funcional de asalariados por voluntarios o por trabajadores informales, o aún no poseen subsidios para considerar la posibilidad de que un supuesto aumento de la complejidad de la profesionalización institucional de esas organizaciones se lleva a un número menor de personas a desempeñar trabajos que antes necesitarían ser gestionados por una mano de obra más extensa (no sólo porque los datos presentados y reproducidos por nosotros hasta entonces son de una estadística descriptiva, sino también porque hacen un puente demasiado estrecho entre el porte de la institución y el número de funcionarios que posee, puente esta que buscaremos complicar a continuación).

Los datos recogidos por el IBGE, desde la década de 1990, muestran que las FASFIL tuvieron un crecimiento del 215\% de 1996 a 2005, dado que las coloca como el sector que más creció en Brasil por estes años, en medidas proporcionales. El "boom" de las ONGs aparece bien datado en las encuestas, siendo éste de la década de 1990. No estamos diciendo que no hay crecimiento significativo del número de entidades entre las encuestas de los años 2002 a 2005, pero ese crecimiento, el orden del 22,6\% (IBGE, 2016, p. 46), se vuelve más tímido a través de los datos de la década de 1990. Esto no nos parece extraño a considerar que el "boom" de la década de 1990 tuvo contextos locales y globales característicos, a ejemplo de la dimensión adoptada por la ECO92; de la difusión de información sobre las agencias de cooperación internacional; de las Reformas Administrativas en el País; de la creación de entidades ligadas a preocupaciones de género o ambientales y que actuaban no sólo ligadas a movimientos sociales; de la posibilidad de 
diferenciación entre los múltiples tipos de ONG, etc; lo que genera más visibilidad al sector, que crea elementos que estimulan un ciclo de crecimiento. Sin embargo, no tenemos en cuenta que las propias limitaciones (y aquí también hablamos de cierta saturación de ese "boom") del sector auxilian la baja secuencial de crecimiento, sobre todo, cuando muchas organizaciones no alcanzan las condiciones necesarias para el mantenimiento y terminan por ser ejemplos de reelaboración de ideas en el universo de las ONGs.

Destacamos que esas características no son las únicas que se mueven en el gráfico de caída numérica del surgimiento de las $\mathrm{ONG}$, primero, porque aunque ese número se ha reducido a partir de los datos de 2002, es aún positivo, apunta un crecimiento y en segundo, porque las proposiciones esbozadas aquí no son de un todo explicativas a esta superficie de investigación cuantitativa del IBGE, pues no tendríamos subsidios para decir qué variables se sustentarían en esos modelos de la investigación del Instituto en un supuesto análisis de regresión múltiple, un plan inferencial cuantitativo con esos datos.

La referida investigación "Las fundaciones privadas y las asociaciones sin fines de lucro en Brasil", que utilizamos como un referencial explicativo-descriptivo, nos dio un punto sólido para hablar de un recorte espacial de ONGs en Brasil, y/o llegar a los planes más específicos de las organizaciones en el Nordeste. También nos hace cuestionar los propios formatos de las encuestas sobre las ONGs del País, sobre todo, las más amplias y que funcionan como base para otras investigaciones que se publican periódicamente en el sector y que tienen sus resultados utilizados a un alcance, principalmente, de ONGs que allí ven un retrato de sí, por donde pueden operar a partir de tales datos. Sin embargo, lo que nos hizo tomar por base esta investigación del IBGE no fue sólo el hecho de ser la más completa, o del gran Instituto investigativo nacional, sino también por los mecanismos de métodos y técnicas que orientó el estudio.

\section{PRESENTACIÓN DE LAS ORGANIZACIONES NO GUBERNAMENTALES}

Como sería inviable tratar sobre esta discusión en todo Brasil, la investigación tuvo como primer recorte espacial la Región Metropolitana de Recife-PE, en nordeste del País. En un primer momento, realizamos una investigación exploratoria sobre la Misión y la Profesionalización de las ONG. Optamos por construir el corpus de la investigación con dos ONGs, ONG 1 y ONG 2, que poseían el mismo foco de actuación, que tenían misiones similares (asistían a jóvenes en situación de vulnerabilidad social) y con complejidades de 
profesionalización institucional distintas, a fin de entender cómo diferentes ONGs se ocupan del mismo tipo de problema social.

Organización No Gubernamental 01:

Pequeña entidad ubicada en Várzea, antiguo barrio de Recife-PE, fundada en 1981 por religiosas de un colegio católico privado con la ayuda de residentes locales. Hermana Fatinha, entonces salesiana de ese colegio, realizaba trabajos comunitarios con niños de la calle y/o en situación de vulnerabilidad social. Al percibir que podría ampliar su trabajo y formalizarlo en una institución, pasó a realizar reuniones con los habitantes de la localidad, en plaza pública, en el refugio religioso, o incluso en las calles, ya que hasta entonces no había ninguna dirección de la institución para la realización de los proyectos soñados. A partir de esas reuniones a principios de la década de 1980, con el apoyo de la actual presidenta de la institución, que en aquella época era profesora del Departamento de Química de la Universidad Federal de Pernambuco, la Hermana Fatinha logró, por medio de donaciones, un pequeño espacio - muy precario - para el surgimiento de la Sociedad ${ }^{4}$.

En 1984, tres años después de la idea inicial, hermana Fatinha falleció. Después de su pretensión inicial, otra religiosa se dedicó a la institución, imaginando un ambiente de socialización, educación y evangelización para los niños, sobre todo, para las niñas. Sin embargo, esta joven de 23 años de edad, recién graduada en odontología, también falleció, dejando en documentación, papeles, que su herencia procedente de Italia debía destinarse a la entidad. Los tristes episodios de dos muertes tuvieron un fuerte impacto en la institución, sin embargo, incluso ante ese cuadro, las demás personas involucradas en la idea inicial, muchas que hasta hoy en la asociación, continuaron dedicándose a la Sociedad.

A partir de este contexto, la institución contó con la donación de un pequeño terreno, hecha por un sacerdote del barrio, que sumada a la herencia recibida dio origen a lo que hoy la organización tiene como espacio físico. Después de esas donaciones, se construyó una casa sencilla. Como saluda la presidenta, la asociación se construyó a partir de donaciones, a menudo aleatorias, a ejemplo de Rotary, con el que la institución tenía un canal de comunicación y cooperación. Hace 32 años, cuando fundada, la ONG no tenía proyectos, incluso por esta todavía no ser una práctica amplia de las organizaciones del tercer sector en ese momento. Aunque la entidad reconoce actualmente que la captación de

\footnotetext{
${ }^{4}$ Todos los nombres mencionados en este artículo son ficticios para garantizar el anonimato de la fuente de información.
} 
recursos se hizo más fácil después de la elaboración de proyectos que se sometieron a varios documentos, no niega que la entidad fue fundada sin una planificación inicial, la Sociedad "vivía con lo que el pueblo daba" (Presidenta de la institución). En los primeros años de existencia la ONG pasó por privaciones extremas, enfrentándose a problemas como la falta de alimentos o saneamiento básico, a partir de las declaraciones de las funcionarias que están en la institución desde la fundación hasta hoy, que en los primeros años de existencia la ONG pasó por privaciones extremas. Estas partes de la historia de la entidad son cotidianamente recordadas por las funcionarias como un contrapunto a la superación y por lo que poseen actualmente.

La institución fue fundada en una perspectiva asistencialista de captación de recursos, pues, necesidades básicas y emergentes como la alimentación de niños, por ejemplo, poseen un tratamiento y valorización diferentes, siendo legítimo, desde el punto de vista de los agentes, el "pedir" para alimentar niños pobres. En esta prerrogativa, aún a principios de los años 1980, Sor Fatinha, fundadora, llamaba a donantes - personas físicas solicitando ayuda financiera, llegando a establecer un carné de pago mensual con la lista de esos donantes / socios asiduos, que sostenían financieramente la entidad.

En 2008, cuando estuvimos por primera vez a iniciar el trabajo de campo que origina estas páginas, la Sociedad se dedicaba al mantenimiento de una guardería con 25 niños para niños de hasta cuatro años de edad que quedaban en la institución por un período de ocho horas todos los días. Paralelamente, la asociación tenía actividades para ocupar niños y adolescentes a partir de, aproximadamente, ocho años de edad, en actividades extraescolares. La idea de este segundo tipo de intervención, con los jóvenes, parte del supuesto de que estos niños y adolescentes, matriculados en escuelas públicas de la región, deben pasar la mañana en el aula, pero que no deben quedarse sin una actividad concentrada por la tarde, debiendo la ONG ocupar el resto del período de esos jóvenes con actividades lúdicas, cursos de teatro, danza, fútbol, música, artesanías etc. En 2010, la guardería fue cerrada por falta de financiamiento, en lo que sus niños fueron trasladados a otras instituciones en la región. Actualmente, la ONG atiende a cerca de 35 jóvenes directamente.

Sobre el cuadro general de cómo se encuentra la entidad actualmente, observamos que es una institución filantrópica con deficiencia de dependencias físicas "sencillas", con cerca de dos "funcionarias" que realizan servicios generales como los de cocinar, cuidar a 
los niños y adolescentes y, mantener la higiene en las dependencias de la institución y un cuadro de aproximadamente diez voluntarios (a contar con la dirección, que es totalmente polivalente y activa en las actividades, siendo la presidenta la misma persona que administra las burocracias y la educación de los niños en los detalles). Señalamos que todos estos agentes citados establecen contratos de voluntariado con la institución, es decir, la entidad no posee funcionarios dentro de las normas establecidas por la formalidad de las leyes de trabajo en Brasil pero ya partir de estos contratos de voluntariado subsidia a los "funcionarios" cuando tienen proyectos financiados por el Estado para que éstos puedan mantener el funcionamiento de la organización. Podemos decir que, de derecho, es una ONG formada por voluntarios, sin embargo, debido al pago en efectivo y aunque esa ayuda de costo que se da a los integrantes sea muy pequeña, el servicio realizado no es entendido por los agentes actuantes de la ONG como voluntariado sólo, pues, para éstos, el voluntariado se caracteriza por la gratuidad de la prestación de servicios. A partir de este reconocimiento, citamos como "funcionarios", de una forma amplia, a los agentes actuantes de esa ONG. A ratificar esta visión del voluntariado compartida en la institución, los únicos considerados como voluntarios son los miembros del directorio que no reciben ayuda de costo.

La dirección de la institución está formada por agentes con curso superior en diferentes áreas: servicio social, psicología, química etc. El cuadro de funcionarios, por su parte, está compuesto básicamente por personas con grados de escolaridad más bajos, de primer o segundo grado, salvando eventuales excepciones, especialmente cuando la existencia de proyectos de financiamiento que logran llevar a la organización profesionales graduados. Una vez que la división de tareas de la ONG no obedece a un modelo cerrado de actuación de los agentes, siendo esos polivalentes en sus funciones, como la presidenta que ejecuta las tareas administrativas simultáneamente a las actividades de educación de los niños, organización y limpieza, o del secretario que pinta las paredes con la ayuda de los niños al mismo tiempo que elabora proyectos, los conocimientos provenientes de la formación de cada uno de los miembros del directorio tampoco son ejecutados sólo en sus áreas específicas de saber. A eso, sumamos que la especialización no se hace clara en la entidad, pues, como hablan los agentes, todos ejecutan diversas tareas, independientemente de la formación académica o de la función que ocupan formalmente en la institución. Organización No Gubernamental 02: 
La segunda ONG analizada, a partir de la selección del corpus de investigación guiada en una intención de estudiar dos ONG con misiones relativamente semejantes y con complejidades de profesionalización institucional distintas, fue una entidad ubicada en el barrio de Santo Amaro, en Recife-PE, Brasil, que atiende un público directo de más de 1500 beneficiarios. Así como la ONG 01, la organización fue fundada en la década de 1980, siendo un poco más reciente que la primera por iniciar sus actividades en 1989, aunque esbozada desde 1986. La ONG, hoy premiada en varios lugares, fue imaginada por tres hermanas que se propusieron a emprender en un trabajo de amparo social a las niñas en situación de calle en la Región Metropolitana de Recife, pues, en la época, no existían programas sociales dedicados a las niñas, sino sólo trabajos de represión, como los de la FEBEM (Fundación Estatal para el Bienestar del Menor), que no provenía de la resocialización de las jóvenes.

A partir del recurso de análisis documental, apuntamos que la institución fue fundada tras la promulgación de la Constitución Federal vigente, de 1988, y en paralelo a las discusiones sobre la creación del Estatuto del Niño y del Adolescente. En un contexto de crisis en Brasil, las autoridades se volvían a las rebeliones de niños presos y no se dedicaban a los problemas de las niñas que estaban en situación de calle, vulnerables al mundo de drogas y prostitución. A partir de entonces una de las fundadoras, con una amplia visión administrativa y con su alto nivel de información y escolaridad, fue a países europeos divulgar la situación de vulnerabilidad social vivenciada por las niñas de Recife. Con el aumento de visibilidad de la institución en el exterior, la ONG logró los primeros financiamientos que viabilizaron la formación de una sede en el centro de Recife.

Con el crecimiento a lo largo de la década de 1990, la ONG amplió su público objetivo, dedicándose a jóvenes entre 7 y 24 años de edad no sólo en situación de calle como también residentes de comunidades de bajos ingresos de la Ciudad y, a partir de 1994, pasó a trabajar con niños y expandió su actuación a las familias de los jóvenes de las comunidades. La entidad realizó una división de trabajo a partir de tres focos de actuación, subdividiéndose en tres programas.

El primer programa, que atiende a niños y adolescentes entre 7 y 17 años de edad, busca la reinserción de los jóvenes a sus familias, a la escuela y a la comunidad a partir de nociones como los derechos de ciudadanía de niños y adolescentes por medio de actividades lúdicas diversas, como teatro y danza. El segundo programa tiene como público 
adolescentes y adultos, de ambos sexos, entre 13 y 24 años de edad, y objetiva la ampliación de los espacios político y social de adolescentes en sus comunidades. El grupo realiza actividades dentro de la institución, como reuniones de capacitación, debates sobre actualidad, sobre la formación de jóvenes en el mundo del trabajo, terapias comunitarias etc. Muchos de estos jóvenes son encaminados al mercado de trabajo por la propia ONG, teniendo en cuenta las asociaciones que esta establece con las empresas. Además de las actividades en la institución, los coordinadores y agentes actuantes trabajan con la noción de educación, capacitando a estos jóvenes para que lleven la información incautada en la ONG a sus comunidades, a partir de reuniones y grupos de concientización que "forman", indirectamente, un grupo mayor de individuos, acompañados por los jóvenes y por los agentes actuantes de la institución, a ejemplo de la coordinadora del Programa, y en articulación con líderes comunitarios.

El tercer programa, orientado a la profesionalización e iniciación al trabajo, absorbe a los jóvenes venidos de los dos primeros programas y capacita profesionalmente a este público con cursos de informática, artesanía, moda, culinaria, telemarketing, manipulación de medicamentos, calidad de ventas, reciclaje, estamparía etc. Sin embargo, aunque el tercer programa se dirige a un público mixto entre 16 y 24 años de edad, ejecuta proyectos como la capacitación de mujeres al mercado de trabajo con cursos de corte y costura, siendo el público de este proyecto compuesto por mujeres de diferentes edades entre 28 y 74 años, aproximadamente. Los proyectos ejecutados por el tercer programa tienen una duración promedio de hasta dos años e involucran disciplinas como nociones de derechos humanos, informática, raciocinio lógico, incentivo a la lectura y otras más. Se observa que estos Programas desarrollan terapia comunitaria entre los miembros de los proyectos, como una actividad en común a los tres programas, siendo ésta una de las filosofías de la institución, constantemente acompañada por profesionales capacitados (psicólogos).

La entidad actúa en una serie de espacios públicos/políticos para ejecutar lo que entiende como lobbying y advocacy. Es miembro de la Asociación Brasileña de Organizaciones No Gubernamentales, es parte del Parlamento Joven del Ayuntamiento de Recife y se articula con proyectos como el Presupuesto Participativo en la búsqueda de la implementación de políticas de derechos humanos, siendo también socio en la Secretaría de Derechos Humanos y Seguridad Ciudadana de la Ciudad de Recife y actúa como socio en frentes gubernamentales y no gubernamentales, a ejemplo del Consejo Municipal de 
Asistencia Social y del Movimiento Nacional de Derechos Humanos. La institución también se moviliza en diferentes foros, redes y secretarías.

\section{DIVISIONES DE TRABAJO, ESPECIALIZACIÓN Y BÚSQUEDA POR PROFESIONALIZACIÓN.}

De acuerdo con los contenidos observados en el análisis de campo y ante la pregunta de partida sobre las consecuencias del actual proceso de profesionalización institucional en las ONGs, buscamos percibir empíricamente los elementos de caracterización de la profesionalización institucional para problematizar las entradas de división del trabajo y búsqueda de profesionalización.

Organización No Gubernamental 01:

La ONG 01 se caracteriza por los individuos que allí están como una entidad en que las relaciones personales de trabajo se dan de forma colaborativa y amistosa. Los papeles desempeñados por los agentes, aunque denominados como "nutricionista", "profesora", "auxiliar de cocina", entre otras categorías, se funden en lo que se entiende por polivalencia de funciones. Una de las razones para este formato de división de trabajo, afirman los entrevistados, está en el hecho de que la entidad no posee recursos de sostenibilidad financiera perennes, lo que la deja como una institución que todo lo que puedan por ella hacer, es bienvenido, es decir, una nutricionista ejercer un papel de profesora de educación física, por ejemplo, es una plusvalía inmediata al problema de la escasez de profesores en esa área. "Toda la gente es buena onda (...) si la persona viene, el personal de la directiva no puede exigir demasiado." (Lourdes, presidenta). El llamamiento por ayuda voluntaria es perceptible en todas las palabras y sobresale cuando por aquellos que están en la asociación por más tiempo, como la presidenta que, mayor que los demás, se dedica a la organización desde los tiempos en que nada había allí de infraestructura o proyectos para apoyo financiero. Sobre las relaciones interpersonales, en frecuencia significativa, cuando cuestionamos sobre este tema, muchas respuestas nos venían en el sentido de que el trabajo para la institución era en favor de una mejora de ésta y, tal mejora, se basaba mucho en aspectos físicos como tener un material de trabajo adecuado para la cocina o incluso un suelo/piso digno a ejercicios pedagógicos: "cuando llegué, aquí atrás, estaba lleno de matorral" (Rosa, servicios de limpieza). A eso, se suma el orgullo a aspectos valorativos claros sobre el trabajo que allí desempeña: "Vi algunas fotos pasadas, era muy fea. La gente 
llega y dice: "Está limpia la ONG 01!" (...) Como tengo ahora en los servicios generales, lo limpio todo, dejo todo arreglado" (Rosa, servicios de limpieza).

Ya en análisis a una de las primeras dimensiones analíticas, sobre los lazos de sociabilidad en el mundo del trabajo, se nota fuertemente que lazos primarios son los pilares de tales prácticas. Bajo la lógica familiar, la fuerte presencia del marido y de una de las hijas de la presidenta se sitúa como única hipótesis de mantenimiento de la organización. "Soy la más cercana a la mamá, a papá y por eso me he donado a ese trabajo..." (Marília, hija de la presidenta, licenciada en educación física). Marília llama a Lourdes por "madre" dentro de la institución, actitud que, aunque naturalizada, gana otra figura cuando comparada al frente con la ONG 02 que veremos a continuación. "Mi misión es personal, ayudar a mi madre aquí. No voy a abandonar a mi madre, independientemente de recibir nada aquí. Entonces, mientras viva, tenga aquí, aquí estoy dentro de mi posibilidad" (Marília, hija de la presidenta, licenciada en educación física). Estas relaciones primarias, en otra perspectiva, proporcionan posibilidades de afecto en la resolución de problemas cotidianos, como subraya Vilma Lima (2004), afecto que puede ser una herramienta en problemas circunstanciales.

En la ONG existe la función de coordinación y fomento de proyectos. Aunque la persona atribuida a eso, auto denominada como una voluntaria que no recibe ningún subsidio para el desempeño de sus tareas, piense en determinados aspectos organizacionales como en posibilidades de elaboración y sumisión de proyectos a ediciones de financiamiento, la presidenta (que vive todos los días en la asociación) es quien termina por estar al frente de éstas y de todas las demás cuestiones, aunque, igualmente, nada reciba en plata por el trabajo al que se dedica intensamente. Se observa que, en una ONG poco burocratizada como esa, rara es la hipótesis de conexión entre los discursos de cómo debería ser la entidad en organizaciones de tareas para lo que realmente es ejecutable frente a limitaciones gerenciales.

Los problemas a los que se dedica la nutricionista polivalente se enfrentan a otros de base, como un niño en edad escolar avanzada no saber escribir el nombre de una fruta en una dinámica grupal de educación alimentaria. Por eso, la lógica de trato familiar, de la percepción del individuo como un todo (aunque no citada en las palabras de las entrevistas, verbalmente, se nota como una constante preocupación cotidiana, del saber quién es la familia de un niño x, como convive como los vecinos, etc.). Sin embargo, esta visión global 
del individuo se relaciona con una lógica inmediatista, algunas veces más amplia, algunas veces menos, lo que termina, al fin y al cabo, menos estratégicas porque cuando se rompe estos lazos primarios (en alguna situación en que las cercanías personales/personalizadas ya no existen) la institución queda sin margen de maniobra, sin opciones.

Se observa que al mismo tiempo que existe la percepción del individuo como integral a los ojos del agente en la organización, recurso que se da en práctica a través de lazos primarios, éstos se limitan ante la mínima dificultad por no estar la entidad amparada por una lógica de la sostenibilidad más universal, perenne. Este aspecto gana luz por el hecho de que la organización no tiene una metodología de trabajo sustancial para guiar las acciones y orientar las tomas de decisión.

Regresando a la división de trabajo, notamos que la polivalencia en estos términos es, pues, una faceta a los aspectos de la especialización. "La gente aquí es un poco de cada cosa (risas): psicóloga, nutricionista...". Sin embargo, no estamos diciendo que ante una caracterización polivalente de funciones los agentes no perciben las posibilidades de división de tareas por competencias, a ejemplo de la función de administración de la cocina que antes era delegada a personas sin formación en el área y que hoy es administrada por una nutricionista que allí estuvo por primera vez a través de una financiación de uno de los proyectos de la entidad y que luego se volvió voluntaria cuando la falta de cualquier dinero que le asegurase una remuneración en la institución. Si la especialización no es una característica frecuente en la ONG 01, aunque existe una estructura de división de tareas y tareas de acuerdo con las funciones que cada individuo ocupa, el hecho de que los agentes sean polivalentes ocurre concomitantemente a la coexistencia de relaciones primarias y secundarias de socialización en el trabajo ya que constatamos que la entidad fue fundada a partir de lazos primarios, incluso habiendo una tímida transferencia de algunos de ellos a lazos secundarios en la medida en que la división y la institucionalización de tareas se interconectan.

Hay una falta expresa de la habilidad de la entidad para abordar problemas específicos, lo que no significa, sobre todo, falta de conciencia de la necesidad de profesionales especializados en el desempeño de determinadas tareas. Lo que termina en la práctica es que, ante imposiciones, la ONG altera lo que el discurso y los moldes de funciones profesionales pueden proponer. La visibilidad para alternativas de crecimiento profesional específicas a los trabajos de los agentes se desvanece frente a necesidades 
urgentes y, aunque en las conversaciones hemos encontrado una constante de que "estudiar y profesionalizar es importante", poco se nota un compromiso en profesionalización personal de los agentes.

A estas cuestiones se asocia la fragilidad en la planificación institucional. La organización envía proyectos al Gobierno del Estado y, cuando son aprobados, los ejecuta, cuando no, queda a la deriva de imponderables, sin una alternativa, un "plan B", para la continuación de las actividades. Un ejemplo de estas limitaciones es un episodio en que, un día, la institución estaba de luto por el fallecimiento de una de las funcionarias y se dio por un día cerrada. En ese mismo día, un corresponsal de asistencia social en la asociación estuvo para hacer una especie de visita de verificación de funcionamiento. Cuando percibió que la organización estaba con las puertas cerradas, repasó a la información a las autoridades competentes que suspendieron incentivos.

Es perceptible en el campo que los márgenes de maniobra están sujetos a posibilidades restringidas de resolución de impasses. También, que se limitan a las movilizaciones para la captación de recursos, lo que lleva al "esperar" de ayudas. Veamos ahora las experiencias de la ONG 02 en estos aspectos generales.

Organización No Gubernamental 02:

Como hemos visto, la ONG 02 también fue fundada por relaciones primarias a partir de la unión de tres hermanas. Hoy considerada una ONG renombrada y de referencia en las áreas que actúa, tiene el aporte de estas relaciones para la ejecución de los trabajos pero se orienta, sobre todo, en la prosecución de lo que formó de cierta cultura por lazos secundarios en el desempeño de funciones institucionales. Se observa que aunque los agentes puedan desarrollar relaciones de amistad, emocionales, el sustento de lo que hay allí es de relaciones a componer las exigencias para el mantenimiento de las tareas, a ver por la forma de selección de los funcionarios, basada en las competencias que disponen de cada candidato, ya sea para prácticas, para voluntariado etc.

Los entrevistados separan la institución de sí cuando hablan sobre ella. Simbólicamente, en paralelo a lo que dijimos sobre la relación entre madre e hija en la ONG 01, en la ONG 02 una de las fundadoras de la institución, que asume uno de los más importantes cargos de coordinación de la entidad, tiene un hijo que también trabaja en la organización. Entrevistamos a este chico por más de dos veces y sólo por ocasiones esporádicas pudimos detectar que se trataba de un hijo y una madre porque se porta como 
uno de los empleados en ambiente de trabajo, como colegas, y la palabra "madre" tampoco fuera pronunciada (sólo la notamos fuera de aquel contexto de horario de servicio). Lo que puede parecer un pequeño detalle, nos dice no sólo en cómo tales agentes se portan de forma más burocratizada, sino sobre la conciencia de cómo lo hacen en la medida en que hay espacios delimitados para la exposición de lazos afectivos. También no versamos aquí que estos lazos primarios, circunscritos en aspectos emocionales, sean "malos" a los aspectos organizacionales (y aquí una vez más corroboramos con las tesis de Lima, 2004 sobre la importancia de los vínculos primarios para las actividades de voluntariado, por ejemplo). Lo que reflejamos es que la dosificación excesiva de un aspecto u otro termina por comprometer el progreso de las funciones y limitar las posibilidades de maniobra, a ejemplo de la contratación de un funcionario competente de determinada rama en detrimento de la función de otro que en la asociación está por ser simpático o algo del género. En suma, esta habilidad en detener lazos primarios y secundarios suena al presente análisis como un aspecto importante al que se refiere a las divisiones de trabajo.

¿Cómo se organiza el trabajo en la ONG 02? Aunque con rasgos de especialización notablemente más fuertes que los de la ONG 01, la polivalencia no desaparece en esta lógica. La polivalencia se remite a aspectos grupales y, aunque no deja de ser la ejecución de varias acciones distintas de por el mismo agente, se utiliza como estrategia para el mantenimiento de los intereses de la institución.

Por una óptica específica, las líneas de actuación de la entidad, divididas como presentamos, se dan en espacios diferentes y una de ellas actúa directamente en la comunidad, donde montó "filiales" en ciudades de la Región Metropolitana de Recife para estar más cerca de las comunidades y no más concentrada en el centro de Recife. Esta fue una medida balizada no sólo en la posibilidad de estar próxima a las comunidades, sino también en la economía con costos de desplazamiento de los adolescentes o demás atendidos que recibían ayuda de transporte hasta la entidad.

La especialización de funciones es transversal al aspecto de la polivalencia en la ONG. Cuando buscamos saber por parte de los agentes si ellos se consideraban especializados en algo, se saltó, en casi unanimidad de las entrevistas, que eran especialistas en la metodología sistémica de trabajo que tenían, en la manera de orientar las acciones dentro de aquello que el equipo comprende como forma de intervención social responsable. Aunque tal metodología no nos ha sido "explicada" exactamente, notamos, a 
partir de un análisis temático puntual de las entrevistas y de los materiales de publicidad de la organización, que es una suma de los aspectos que aquí puntuamos que involucra los planes administrativos y los márgenes la maniobra de la organización, de colocarse como protagonista de las escenas que rondan la realidad donde están insertadas en el nordeste de Brasil.

Buscamos percibir el compromiso de los agentes en formaciones más allá de sus originales/profesionales, o sea, si la metodología propuesta por la entidad estaba anclada en expertise específica al profesional del tercer sector. Para nuestra sorpresa, lo que encontramos fue el intento de articulación interdisciplinaria, en que un agente transporta a otras funciones y éste, a su vez, potencializa posibilidades de que el solicitante desarrolle otras salidas de resoluciones de problemas cotidianos. Los entrevistados también recurren a cursos y formaciones sobre captación de recursos, marketing, relaciones humanas para el tercer sector etc en la mayoría de las veces ministrados por otras ONGs en la búsqueda de una formación más adaptada al trabajo desarrollado, sin embargo, más que eso, se destacó en las entrevistas la capacitación interdisciplinaria de trabajo.

En el caso de las relaciones entre las personas que actúan en pro de un objetivo común (KRIEGER, 2001), la percepción de campo de cada uno de los elementos esbozados, como la especialización o los lazos primarios presentes en las asociaciones, sólo tiene sentido cuando se piensa conjuntamente porque los agentes no disocian tales aspectos en la práctica de las acciones (la toma de decisión sobre uno de estos puntos gana significado sólo cuando se piensa a partir de los demás).

El concepto de organización propuesto por Bernoux (1998) prima por una construcción de organización a partir del intento de explicación de los comportamientos individuales dentro de las instituciones. Esta fue una herramienta importante en el campo para delimitar el objeto en el diseño empírico. Buscamos a partir de esto crear un análisis por la observación de frenados grupales, como las presentadas en la ONG 01, para indicar las "burbujas de gas" de la organización frente a las salidas encontradas por otra que, dentro de diferentes contextos, posee elementos comunes que las hacen percibir como ONGs en la ciudad de Recife. Lo que buscamos aquí de la colaboración de Bernoux fue aislar al agente de la estructura para analizarla y, intentamos ir más allá, haciendo posteriormente el camino inverso de por el agente rondar los problemas estructurales. Fue importante percibir en campo la abstracción del elemento "organización" que nos anticipaba Bernoux, sobre todo, 
cuando las funciones de tales individuos en el organigrama de la institución (ONG 02) y de la polivalencia que se presentó a los dos casos. La especialización es interesante en las organizaciones en contextos puntuales localizados y es solicitada por agentes insertados en los trabajos polivalentes. Igualmente, cuando la presencia acentuada de polivalencia en algunas situaciones, se nota un desequilibrio en la estructura de estas funciones que, a su vez, afectan la estructura más amplia de la ONG.

Fue también en campo que pudimos verificar la síntesis de Ferreira (1996) que proponía una tipificación ideal de organización: "1. Desarrollar el uso masivo de las inteligencias a todos los niveles jerárquicos e incrementar las capacidades para lidiar con el complejo y con el evolutivo" (FERREIRA, 1996, p. 274). Este punto fue verificado en agotamiento en las dos organizaciones y materializado en lo que decimos arriba sobre la polivalencia de funciones. 2. "Adoptar una filosofía de gestión caracterizada por la elevada calidad de las relaciones individuales y la cooperación entre funciones" (FERREIRA, 1996, p. 274), complementa a la primera idea y refuerza que encontramos en el campo, no sólo a partir de la observación directa, sino también de las palabras en las entrevistas, que la división del trabajo se da de la forma más horizontalizada posible. Sin embargo, en la ONG 02 se evidencia que aunque las relaciones tienden a ser horizontalizadas, se pautan en divisiones de tareas y agendas menos porosas en las que aunque los agentes de directorios sean, por ejemplo, accesibles a los demás, se pone más clara a división de funciones que la ONG 01. 3. "Asegurar la calidad de las decisiones no sólo estratégicas, sino también de las múltiples y pequeñas decisiones, acortando las líneas jerárquicas y descentralizando competencias hasta la base de la pirámide", aquí Ferreira (1996) tece sobre la "calidad" de las decisiones, sin embargo, en campo notamos que la calidad de cualquier opción es relativa a los demás factores que ligan la estructura, o sea, decir que el cumplimiento de un objetivo x es lo que hay de más importante sólo lo es en sincronía con los demás. Ejemplo: aumentar el número de niños atendidos en la ONG 01 sólo tiene sentido si las que ya allí están alimentadas en el período de las actividades. 4. "Evitar la especialización aislada y conferir poder y especialización a través de la multiplicación del trabajo en grupo y de la valorización de los enfoques pluridisciplinares" (FERREIRA, 1996, p. 274), como se ha visto, no nos encontramos con intentos de especialización aislada en ninguno de los casos, corroborando con la tipificación de Ferreira (1996). 5. "Estimular una política de implicación de las personas, a través de la creación y el mantenimiento de un proyecto 
movilizador capaz de atraer y entronar vencedores, y de innovación en los sistemas de recompensa y de reconocimiento" (FERREIRA, 1996, p. 274), perspectiva no encontrada en ninguno de los casos, sobre todo, porque las entidades ya se insertan en dos extremos que son los problemas de sostenibilidad financiera y también porque los reconocimientos ya se introducen implícitamente en las relaciones primarias de forma menos materializada de análisis. En suma, estos elementos presentados pasan por las perspectivas de poder, tareas y papeles presentados.

$\mathrm{Al}$ asociar estos modelos empíricos a teorías pragmáticas de las organizaciones, notamos que el ejercicio más grande pretendido en ambas entidades es el de adaptarse a los nuevos modelos sociales. Este parece ser el motivo por la carrera de gestión. La profesionalización institucional es sentida con más fuerza en la ONG 02 debido a las posibilidades de reordenamiento organizacional más claras que posee $\mathrm{y}$, consecuentemente, por crear nuevos modelos de gestión de acuerdo con las exigencias de los editales a los que se somete, creando una agenda mínima a atender diferentes demandas. La normativa para estas acciones está presente al mismo tiempo que innova y sorprende con la "creatividad" que las ONGs tienen para resolver ciertos problemas, a ejemplo de cuando funden proyectos interdependientes ante la financiación de uno solo. Tal normatización no aparece a las ONG como un cierre de reducto porque las organizaciones pueden funcionar como un sistema abierto, elemento que se refiere a la autonomía de las asociaciones. Como se ve por Mintzberg (2003), cuando no existe una conciencia integrativa entre las dimensiones analíticas que componen las instituciones, la entidad no funciona de forma eficaz. Sin embargo, contrariando a Mintzberg en alguna medida, nuestro campo no encontró dimensiones analíticas o categorías estancas, sino mucho híbridas, lo que fomenta la complejidad institucional. ¿Y qué nos hace decir a priori que la ONG 02 funciona mejor que la ONG 01 en términos organizativos/operativos? En esa, tales dimensiones se presentan como más coherentes en un plan de acción común, cuando en la ONG 01 estos elementos se contradicen con mayor frecuencia.

\section{ASPECTOS QUE CARACTERIZAN UN ESCENARIO EN MOVIMIENTO}

Hablamos de las dimensiones analíticas indicadoras de la complejidad de profesionalización institucional a partir de dos estudios de caso. En la ONG 02, encontramos una compleja división de trabajo, subdividida en diversas áreas de actuación; 
alto nivel de especialización en las actividades desarrolladas; pirámide jerárquica consolidada; relaciones de trabajo no reducidas sólo a las relaciones primarias de sociabilidad; articulación en redes; estrategias de planificación para el futuro de la organización; utilización de la Misión en la captación de recursos etc. En la ONG 01 no se encontraron tales indicadores con la misma fuerza, como ya se ha demostrado. De esta forma, entendemos que la ONG 01 es una entidad de complejidad profesional menos expresiva en comparación con la ONG 02. Igualmente, hablamos de temáticas transversales a la profesionalización institucional considerando, especialmente, la perspectiva de los agentes frente a determinados elementos variables en las ONGs.

Nos enfocamos ahora en sus cosmovisiones por lo que se refiere directamente a la profesionalización institucional que estudiamos y que sostiene la pregunta de partida de nuestro trabajo. En principio, la percepción de profesionalización en la ONG 01 no gana contornos materializables en la medida en que, en las entrevistas, los agentes no conectan el contenido de la palabra "profesionalización" ni bajo un enfoque más institucionalizado, tampoco a un enfoque más personalizado, o sea, no se percibían como parte de un proceso de profesionalización del tercer sector. Sin embargo, esto no significa que no comprenden por sus trabajos aspectos que se refieren a la profesionalización institucional o que no sienten los efectos de ser, y aquí adelantamos, menos burocratizados y portadores de una profesionalización menos compleja del tercer sector. En un ambiente en el que las relaciones primarias y la racionalización sustantiva abren las estructuras relacionales, la perspectiva de la agencia sobre el fenómeno de la profesionalización tendería a variar según los papeles que los agentes desempeñan fuera de la organización. Sin embargo, y justamente por ser de ese tipo de socialización y racionalización, aunque los agentes de la ONG tienen acceso a un mundo material privado privilegiado en relación a los niños a quienes prestan servicios, circunscriben aspectos humanizadores de sus vidas particulares en los contextos en que trabajan que son no sólo contextos de pobreza, sino también de "proyecto", "cocina", "ropa por lavado" etc. Por lo tanto, el primer aspecto que les salta a las palabras es el de la multiplicidad de campos que integran (y una vez más, de la polivalencia) que aunque presente en ONGs del modelo 02 , son más vivos e inmediatos en el 01. En la ONG 02, encontramos una ramificación programática de lo que puede ser la profesionalización institucional y la búsqueda de esa profesionalización como la manera de escapar a los problemas de la sostenibilidad. 
Al seguir alrededor de la perspectiva de los agentes en la ONG 01, los puntos que más resaltan sobre los elementos globales que creen girar en torno a la profesionalización son: 1. no se consideran profesionalizados porque no poseen una estructura física suficientemente adecuada, aunque ya ha sido mejorada con el paso de los años; 2 . no tener profesionales contratados, en lo que estos profesionales, con conocimientos específicos y donde trabajar con responsabilidad, podrían proveer una burocratización acertada a posibilidades de financiación.

Cuando preguntamos a los agentes de las ONGs lo que entienden por profissionalización, en la ONG 02 encontramos agentes que consideraban a la entidad como profesionalizada y, otros, que no. El más curioso fue que estos agentes localizan la profesionalización a posiciones y contextos, es decir, sólo bajo determinadas condiciones la institución es considerada profesionalizada. Estas condiciones están fuertemente relacionadas con la sostenibilidad financiera en la medida en que atribuyen parte de los problemas de la sostenibilidad a una supuesta falta de profesionalización del tercer sector como un todo y del no enfrentamiento social de sus condiciones frente a los demás sectores. A estos demás sectores, notadamente al Estado, se acreditan las "culpas" de la inestabilidad. Sin embargo, se observa también que la profesionalización en el caso de la ONG 02 no ha concluido consistentemente que llegue con el conocimiento profesional (a través de un agente portador de competencias, o de diploma). Es ella, para los agentes, una construcción de ese conocimiento. Esto refleja procesos inherentes a la formación de la lógica de trabajo interno en lo que reflexionar sobre la lógica de la profesionalización institucional ya es un indicador empírico de complejidad de esa profesionalización.

En concreto, la poca intimidad con el término "profesionalización" en la ONG 01 es también demostrativa de que sus agentes no se insertan con frecuencia en debates sobre el tercer sector, en que talleres y cursos dictan constantemente sobre el tema, a diferencia de la ONG 02 que, incluso, sedia eventos como éstos. Además, los problemas de la profesionalización institucional son datados, contextuales y flexibles, en lo que hay que estar atento a cambios sociales más allá de las ocurrencias internas a una organización particular. Al investigar sobre las relaciones que hacían los agentes de la ONG 01 sobre la entidad y las coyunturas políticas/económicas, tuvimos poca respuesta conectando los dos mundos, en lo que entendemos que tales agentes no relevan las crisis financieras, los cambios de gobiernos entre otros acontecimientos como limitadores o fomentadores de 
financiación, a ejemplo de la ONG 02 que sitúa la crisis económicas o los marcos políticos como signos considerables en la toma de decisiones.

En la ONG 02 tenemos una tendencia expresiva de reflexiones sobre los contextos políticos/financieros externos a la institución que son acompañados como medidas estratégicas a los planes de gestión. Es decir, las perspectivas de los agentes sobre el futuro de la organización están enlazadas, sobre todo por la dirección y las coordinaciones de los Programas, a un seguimiento de la economía mundial ya las tendencias de nivel macro del Tercer Sector en el País: "Veo muy grande el futuro de la ONG, por una tendencia global. La búsqueda de proyectos es constante" (Carmem, entrevistada). Las nociones de continuidad de las actividades sufren, en gran medida, influencia de la formación escolar y del mantenimiento de búsqueda por conocimientos sobre la relación entre los tres sectores de la sociedad, o sea, del constante reciclaje de los entrevistados, de sus trabajos basados en discusiones, como en grupos de formación profesional, lo que refleja inmensamente en la administración de la ONG y en la construcción de un modelo organizacional que sustenta las actividades de la entidad. La perspectiva de futuro de la ONG 02 está ligada por los agentes a posibilidades administrativas al mismo tiempo que se tocan en discusiones holísticas sobre el papel del tercer sector, en las posibilidades de que las ONG deberían nacer para morir al cumplir su papel en la sociedad. En el marco de la investigación de campo (división del trabajo porosa entre las funciones ejercidas por los miembros, poca especialización, poca flexibilidad para la captación de recursos, relaciones primarias sobrepuestas a las relaciones secundarias de socialización en el trabajo, bajo poder de planificación para el futuro de la entidad, no inserción en redes de cooperación con otras instituciones, entre otras) averiguamos que la entidad tiene poca complejidad de profesionalización institucional como ya mencionamos e incluso esta característica influye y es influenciada por el hecho de que la ONG haya poca visibilidad dentro del Tercer Sector.

Otra característica referente a la no expresiva complejidad en la ONG 01 reside en problemas gerenciales en el área pedagógica, presentes en el ejemplo de que algunos niños y adolescentes no les gusta participar en determinadas actividades y generan una falta de cohesión en el grupo, dispersión y no interacción, algo de los profesores y del directorio de la ONG. Cuando se le preguntó sobre este punto, la dirección tendía a respuestas de que ese problema era antes ligado a características personales de las niñas y que no se podía exigir 
más disciplina o aplicar otro método pedagógico que generaría desinterés de esas por la entidad, pues perderían su público y, consecuentemente, dejarían de ayudar y participar en la vida de esas jóvenes. Lo que no significa que haya falta de interés por parte de los agentes en solucionar tal problema, sin embargo, no localizamos en la organización un aparato pedagógico de acción que podría ser traído, por ejemplo, por profesionales especializados en esta área educativa.

A partir de trechos como éste, visualizamos la "transferencia" de la posición de la presidenta para la madre (esfera familiar) o para la batalladora que logra por su esfuerzo de sostener la organización 01 y mantener las puertas de la entidad abiertas en medio de todas las dificultades encontradas. Este modelo presume concentración de actividades en un único miembro, que ha estado presente en la institución a lo largo de toda su historia y, con ello, cuestionamos hasta qué punto esta relación favorece la continuidad de las actividades de la ONG. En algunas entrevistas, desde los funcionarios hasta la dirección, muchos alegaron que, en el futuro, la institución debería estar en las "manos de la comunidad", que ésta debería asumirla. Sin embargo, la forma de llegar a esta etapa de desarrollo democrático de la entidad no fue mencionada en ninguna ocasión. En especial, dos profesoras/directoras no sabían responder cuáles eran las principales dificultades encontradas por la organización: "Creo que quien podría responder eso para usted es Lourdes. Que ella es la batalladora hoy en día. ¡Ella es quien asume!" (profesora entrevistada en la ONG 01). Tales palabras se enlazan a la problemática de la falta de planificación a medio y largo plazo encontrada en la entidad. El futuro de la organización no es visto por los agentes sin la presencia de la presidenta y eso ocasiona preocupaciones entre los funcionarios al notar que no hay quien pueda sustituirla de la misma forma, es decir, en los moldes de dedicación y trabajo que hacen gestionar las actividades desarrolladas por la organización. Al mismo tiempo que la cocinera de la entidad dice que fue a trabajar en la institución por gustar mucho de la presidenta, por ser una buena persona, surge por parte de miembros de la directiva cuestionamientos como ¿En el futuro quién queda? La gente ya habló, pero ... ".

La ONG 02 tampoco se exime de problemas que se refieren a la profesionalización institucional aunque, como dijimos, tenga un marco más complejo a este punto. Uno de los agentes, Vinícius, nos cita los obstáculos burocráticos de la organización que, contradictoriamente, se suman al exceso de flexibilidad que se da a la entidad según los 
plazos que deben cumplir ciertas tareas (no cumplimiento de deadlines). Por otra parte, localiza las redes de cooperación que la institución protagoniza como espacios de la sociedad civil necesarios, indicadores de profesionalización de las acciones, así como el uso del voluntariado para la capacitación del trabajo en red.

Puntuamos lo que nos rindió en términos de análisis de datos la oportunidad de estudio longitudinal en las ONGs 01 y 02 . Esencialmente, pudimos confirmar las tendencias al plan de la profesionalización institucional en las dos entidades. Las dimensiones analíticas que en 2008 nos indicaban categorizar las dos asociaciones se pusieron más firmes a la lectura de las dos realidades en 2012 y cuando volvemos a las organizaciones en otros momentos hasta 2021. Cambios a lo largo de los años existieron en ambas, pero ninguna a cambiar las tendencias apuntadas en un primer instante, a mantener las dimensiones de patrones de investigación que sostienen la investigación. Los objetivos específicos trabajados como soporte para pensar sobre la profesionalización institucional se pusieron en relevancia a la lógica investigativa, así como a la percepción de ejes ejemplificados en los tipos de relaciones y racionalizaciones dentro de las entidades transversales e inherentes a cualquier intento de reflexión sobre la profesionalización institucional.

\section{CONSIDERACIONES FINALES}

La pertinencia de la investigación empírica a partir de los estudios de caso trabajados sobre el objeto de estudio de las ONGs recayó en el hecho de que el tercer sector fue reconocido como una realidad actual, concreta y en transformación. Por lo tanto, volviendo a nuestra pregunta de investigación, ¿cuáles son las consecuencias del actual flujo de profesionalización institucional? Las consecuencias son que con las actuales demandas, cada vez más similares a los modelos de mercado, las instituciones que no se adhieren a un cumplimiento que articule mínimamente las dimensiones analíticas de que tratamos a lo largo de esta investigación, se desvanecen y quedan a merced de mecanismos paralelo. Estos mecanismos, en vez de resolver parte del problema social (de la Misión) a que se proponen, terminan por crear otros problemas igualmente sociales, aparentemente fuera del tercer sector, como la cuestión del empleo de los agentes que quedan inestables mediante los problemas de financiación. Esto que refleja, por supuesto, en el cumplimiento de la Misión al mismo tiempo que la exigencia de profesionalización del sistema crea 
problemas que sólo pueden ser resueltos con más profesionalización y burocratización. Esta consecuencia, dispuesta a un efecto dominó, llega a las piedras de que gran parte de las ONG afectadas por esta lógica, en lugar de dedicarse a los problemas originales que les dan sentido, terminan por dedicarse abundante a los problemas de sostenibilidad financiera, crear en los agentes una nube de inseguridad a las perspectivas de trabajo individuales en vez de allí ver un apoyo institucional por un sistema que tendería a ser racionalmente coherente con la realidad de expansión del tercer sector. Es decir, la profesionalización, aunque portadora de méritos organizacionales, no puede dar cuenta de los problemas que ella misma trae consigo. Crea problemas que ella misma no puede resolver en el marco en que se plantea.

Finalmente, detectamos por las entrevistas analizadas un pesimismo colectivo de los agentes sobre el futuro de las organizaciones, que saltó notablemente cuando se hizo la pregunta sobre cómo veían el futuro de la organización a la que pertenecían. Esto trae una contradicción actual del tercer sector que, si bien propone motivaciones para los diversos problemas sociales que cubre sus misiones, tiene una agencia que, aunque comprometida, está desmotivada y desanimada, especialmente porque aspectos de la vida personal de estas personas son igualmente vulnerables en ante las incertidumbres del sector. Percibimos, por tanto, una agencia frágil en relación al amplio proceso de profesionalización institucional, aun cuando se involucre en procesos de empoderamiento académico personal. Darnos cuenta de estas conclusiones nos parecía importante, pero la relevancia de lo que hablamos está en el proceso que sustenta y genera este trabajo, que cuestiona la forma en que se viene dando y renovando el actual proceso de profesionalización institucional, carente de una regulación práctica y arraigada. en reflexiones que alimentan no solo el mantenimiento de un sistema del tercer sector, sino la coherencia entre éste y los que forman parte de él.

\section{REFERENCIAS BIBLIOGRÁFICAS}

AGUIAR, Andson; MARTINS, Gilberto. A teoria das estruturas organizacionais de Mintzberg e a gestão estratégica de custos: um estudo nas ONGs paulistas. Cont. Fin. USP. Edição Comemorativa, 2006.

BERNOUX, Phillippe. A sociologia das organizações. Porto: Rés, 1998. 
BRASIL. 2006. Constituição Da República Federativa Do Brasil [1988]. Texto consolidado até a emenda Constitucional $n^{\circ} 48$ de 10 de agosto de 2005. Rio de Janeiro, Escala.

CARVAlHO, Cristina Amélia Pereira de. "Preservar a identidade e buscar padrões de eficiência: questões complementares ou contraditórias na atualidade das Organizações NãoGovernamentais?” In: Revista do GENEIT/PPGA/UFRGS. 24p, 1999.

COSTA, José Ricardo Ferreira da. Sociedade Civil, Humanitarismo e Utilitarismo: um estudo empírico sobre os padrões de solidariedade das ONGs da RMR. Dissertação de Mestrado. Recife / UFPE, 2004.

FERREIRA, J. M et.al. Psicologia das Organizações. Portugal: McGraw-Hill, 1996.

HADDAD, Soraia. "A profisssionalização chega às Organizações Sociais”. In: Gazeta Mercantil, 2002.

HILL, W.A e Egan, D.M. Readings in organization theory: A behavioral approach. Boston: Allyn and Bacon, 1967.

IBGE. Instituto Brasileiro de Geografia e Estatística. Ministério do Planejamento, Orçamento e Gestão. As fundações Privadas e Associações sem fins lucrativos no Brasil. IBGE, Gerência do Cadastro Central de Empresas. Rio de Janeiro: IBGE. (Estudos e Pesquisas. Informação econômica), 2016.

KRIEGER, Mario. Sociologia de las organizaciones: uma introducción al comportamiento organizacional. Buenos Aires: Prentice Hall, 2001.

LAKATOS, Eva Maria. Sociologia Geral. 5. ed. São Paulo: Atlas, 1987.

LANDIM, Leilah. Para Além do Mercado e do Estado? Filantropia e Cidadania no Brasil. Rio de Janeiro: ISER, 1993.

LIMA, Vilma Soares de. Dádiva e voluntariado: ações de apoio junto a portadores de câncer. Recife, Programa de Pós-Graduação em Sociologia / UFPE. Dissertação de Mestrado, 2004.

LIMA, Diogenes Fagner de. Entre "raças", cotas e ONGs: uma crítica do antirracismo social-liberal no Brasil. Universidade Federal do Rio Grande do Norte. Dissertação de Mestrado, 2020.

MINTZBERG, H. (2003). Criando Organizações Eficazes: estrutura em cinco configurações. Atlas, 2003.

MELO, Marina. "Panorama da Profissionalização do Terceiro Sector em Portugal". In: Ricot Journal - International Journal on Working Conditions. n. 5, 2013. 
MELO, Marina. “Aspectos Preliminares da profissionalização de ONGs: contextos, práticas e atores”. In: Revista O Público e o Privado, n 36, 2020.

PAES, José Eduardo. Fundações, Associações e Entidades de Interesse Social. São Paulo: Forense, 2019.

RIBEIRO, Mário; MARQUES, Sofia. Uma leitura das ONGD portuguesas no início do século XXI: trajectória e dinâmicas. In: Revista Lusotopie, n. 1, 2002.

SELZNICK, Philip. T. V. A. and the Grass Roots. California Univ. Press. Berkeley, 1949.

TENÓRIO, Fernando G. Gestão de ONGs: principais funções gerenciais. Rio de Janeiro: FGV, 2015.

VIEIRA, Larissa et al. "Responsabilidade social coorporativa e a relação entre ONGs e corporações: uma revisão sistemática da literatura”. In: Revista Engema. N. 01, 2020. 